\title{
A robust xenotransplantation model for acute myeloid leukemia
}

\author{
PV Sanchez ${ }^{1,3}$, RL Perry ${ }^{1,3}$, JE Sarry ${ }^{1,3}$, AE Perl $^{1}$, K Murphy $^{1}$, CR Swider $^{1}$, A Bagg ${ }^{1}$, JK \\ Choi $^{2}$, JA Biegel ${ }^{2}$, G Danet-Desnoyers ${ }^{1}$, and M Carroll $^{1}$ \\ ${ }^{1}$ Department of Medicine, Division of Hematology and Oncology, University of Pennsylvania, \\ Philadelphia, PA, USA \\ 2Department of Pathology, Children's Hospital of Philadelphia, Philadelphia, PA, USA
}

\begin{abstract}
Xenotransplantation of human acute myeloid leukemia (AML) in immunocompromised animals has been critical for defining leukemic stem cells. However, existing immunodeficient strains of mice have short life spans and low levels of AML cell engraftment, hindering long-term evaluation of primary human AML biology. A recent study suggested that NOD/LtSz-scid IL2R $\gamma c$ null (NSG) mice have enhanced AML cell engraftment, but this relied on technically challenging neonatal injections. Here, we performed extensive analysis of AML engraftment in adult NSG mice using tail vein injection. Of the 35 AML samples analyzed, 66\% showed bone marrow engraftment over $0.1 \%$. Further, $37 \%$ showed high levels of engraftment (>10\%), with some as high as $95 \%$. A 2-44-fold expansion of AML cells was often seen. Secondary and tertiary recipients showed consistent engraftment, with most showing further AML cell expansion. Engraftment did not correlate with French-American-British subtype or cytogenetic abnormalities. However, samples with FLT3 mutations showed a higher probability of engraftment than FLT3 wild type. Importantly, animals developed organomegaly and a wasting illness consistent with advanced leukemia. We conclude that the NSG xenotransplantation model is a robust model for human AML cell engraftment, which will allow better characterization of AML biology and testing of new therapies.
\end{abstract}

\section{Keywords}

AML; NOD/SCID/gamma chain deficient; xenotransplantation

\section{Introduction}

Acute myeloid leukemia (AML) is a disease resulting in an uncontrolled proliferation of functionally immature hematopoietic cells with an increasing incidence in adults 65 years and older. Although effective molecular therapies exist for chronic myeloid leukemia and acute promyelocytic leukemia, no single therapy has been identified to treat the majority of patients affected with AML. The majority of AMLs are genetically diverse and, therefore, present a challenge to develop targeted therapies. Therefore, there is a critical need to

\footnotetext{
(C) 2009 Macmillan Publishers Limited All rights reserved

Correspondence: Dr M Carroll, Department of Medicine, Division of Hematology and Oncology, University of Pennsylvania, BRB2/3, 421 Curie Boulevard, Philadelphia, PA 19104, USA. carroll2@ mail.med.upenn.edu.

${ }^{3}$ These authors contributed equally to this work.

Supplementary Information accompanies the paper on the Leukemia website (http://www.nature.com/leu)

Conflict of interest

Dr. Carroll has received research support from Sanofi Aventis Corporation.
} 
develop robust animal models to study the complex biological processes associated with a developing leukemia in vivo.

Non-obese diabetic mice with severe combined immunodeficiency (NOD/SCID) have been used with success to study morphological and biological characteristics of human AML in vivo. ${ }^{1-3}$ However, the level of AML engraftment in NOD/SCID (NS) mice is frequently modest with typical engraftment levels of $0.1-5 \%$ of the mouse bone marrow. ${ }^{1}$ In addition, prolonged engraftment of leukemic cells in this breed of mice was limited by the development of spontaneous thymic lymphomas and a reduced life span. ${ }^{4,5} \mathrm{New}$ strains of mice engineered with targeted deletion of the $\beta_{2}$-microglobulin gene within an NOD/SCID background (NSB) have resulted in models with defects in MHC class I expression and decreased natural killer (NK) cell function that are better suited for studying the progression of diseases such as AML. ${ }^{6}$ However, recent reports have showed that a targeted deletion in the $\gamma$-common chain in NS mice (NOD/LtSz-scid IL2R $\gamma$ c null (NSG)) results in the severe impairment of B, T, and NK cell function and provides an improved environment for growth and development of human cells. NSG mice also have an improved life span of $>90$ weeks and are not prone to develop thymomas. ${ }^{7}$ One earlier report has suggested that craniofacial neonatal injection of NSG mice with primary AML cells could lead to high engraftment, but extensive characterization of the model with the more commonly used intravenous (IV) injection methodology has not been described. ${ }^{8}$

This report describes a comprehensive analysis of the engraftment of 35 primary human AML samples in the NSG mouse strain. For these studies, we injected unsorted leukemic cells obtained by leukopheresis from AML patients consisting of at least $80 \%$ blasts into recipient NSG animals. Similar to the NS model, approximately two-thirds of samples engrafted, however, the level of engraftment was significantly higher and ranged up to $90 \%$ of the mouse bone marrow. Bone marrow and spleen were the primary sites of leukemic cell infiltration among the specimens with the highest levels of engraftment (above 10\%). Unlike earlier reports, we observed limited peripheral blood engraftment in only the robust AML samples that engrafted. Secondary and tertiary transplants of leukemic cells into NSG mice retained phenotypic characteristics of the original sample and were significantly expanded in comparison to earlier described immunocompromised animal models engrafted with human leukemic cells. ${ }^{1,6}$ Leukemic engraftment was not limited to CD45+CD33+ human cells in a few select AML samples, as cytotoxic T-cell expansion was also seen. Our results provide a thorough examination of the engraftment of a diverse set of leukemic samples and a robust leukemic in vivo model ideally suited for therapeutic studies with the ability to expand and isolate adequate quantities of cells for molecular analysis.

\section{Materials and methods}

\section{Primary cells}

AML samples were obtained from the Stem Cell and Xenograft Core Facility at the University of Pennsylvania School of Medicine. Samples were obtained from patients presenting with AML at the Hospital of the University of Pennsylvania with informed consent in accordance with institutional guidelines. Leukopheresis samples were processed by Ficoll gradient centrifugation and mononuclear cells were frozen in fetal calf serum with $10 \%$ dimethyl sulfoxide and stored in liquid nitrogen. The percentage of blasts was determined by flow cytometry and morphological characteristics before purification. Samples with $>80 \%$ blast cell count were chosen for these studies. French-AmericanBritish or World Health Organization classification and cytogenetics were determined at time of diagnosis by the Laboratory of Pathology and Medicine at the Hospital of the University of Pennsylvania. FLT3/ITD (internal tandem duplication), FLT3 D835, and FLT3 wild-type status in AML samples was determined as reported earlier. ${ }^{9}$ 


\section{Flow cytometry analysis}

CD45-APC (BD 555485), CD33-PE (BD 555450), CD19-FITC (BD 555412), and CD2 PECy7 (BD 335804) fluorescent antibodies were used to analyze leukemic cells before and after injection into animals to determine phenotypic analysis of engrafted cells and percentage of leukemic cell engraftment. DAPI or 7AAD (Molecular Probes, Invitrogen, Eugene, OR, USA) were used to exclude non-viable cells from the flow cytometry analysis using FlowJo software version 8.5.3 (TreeStar, Oregon, USA).

\section{Mice}

NSG mice were produced at the University of Pennsylvania using breeders obtained from Jackson Laboratory (Bar Harbor, ME, USA). Mice were housed in sterile conditions using HEPA-filtered microisolators and fed with irradiated food and acidified water. Transplanted mice were treated with antibiotics (neomycin and polymixin) for the duration of the experiment.

\section{Transplantation of human leukemic cells}

Adult mice (8-10 weeks old) were sublethally irradiated with $250 \mathrm{cGy}$ of total body irradiation $24 \mathrm{~h}$ before injection of leukemic cells. Leukemia samples were thawed at room temperature, washed twice in PBS, cleared of aggregates and debris using a $0.2 \mu \mathrm{m}$ cell filter, and suspended in PBS at a final concentration of 5-10 million cells per $200 \mu \mathrm{l}$ of PBS per mouse for IV injection. Daily monitoring of mice for symptoms of disease (ruffled coat, hunched back, weakness, reduced motility) determined the time of killing for injected animals with signs of distress. If no signs of distress were seen, mice were analyzed 12 weeks after injection except as otherwise noted. For secondary and tertiary recipient animals, a range of 2.5-10 million unsorted human CD45+ CD33+ viable cells from bone marrow and/or spleen of primary or secondary recipients were transferred into individual recipients by IV injection.

\section{Assessment of leukemic engraftment}

NSG mice were humanely killed in accordance with IACUC protocols. Bone marrow (mixed from tibias and femurs), spleen, liver, and kidney were dissected in a sterile environment, flushed in PBS and made into single cell suspensions for analysis by flow cytometry (FACS Calibur, FACS Canto, FACS LSR II-BD Biosciences, San Jose, CA USA) and HEMA3 staining of cytospins (Fisher Scientific, Middletown, VA, USA). Bone marrow, liver, kidney, and partial spleens were fixed in Accustain Formalin Solution 10\% (Sigma-Aldrich, St Louis, MO, USA) and were processed by the Histology Core at the Children's Hospital of Philadelphia. Histologic specimens of mouse bone marrow were decalcified and paraffin embedded, slides were made and stained with hematoxylin and eosin (H \& E), reticulin ( Jones Method), and Masson's trichome stain for assessment of tumor necrosis and fibrosis as described earlier. ${ }^{10}$ In addition, spleen, liver and kidney were paraffin embedded and slides were made and stained with $\mathrm{H} \&$ E. Peripheral blood was obtained after killing through cardiac puncture or by retro-orbital bleeds before killing. Whole mouse blood was stained with human CD45- and CD33-specific antibodies followed by red-cell lysis using FACS lysing solution (Becton Dickinson, San Jose, CA, USA). Leukemic cells in peripheral blood were quantified using CountBright Absolute counting beads (Invitrogen, Carlsbad, CA, USA) and the limit of detection was determined to be 10 cells per $\mu \mathrm{l}$ of whole mouse blood. Red-cell lysis with $0.8 \%$ ammonium chloride solution (Stem Cell Technologies, Vancouver, Canada) was performed before flow cytometric analysis. All images were taken at $\times 50$ magnification using a Nikon $80 \mathrm{i}$ microscope coupled to a Leica DFC320 CCD camera and acquired with Leica FireCam 1.4 Software. 


\section{Immunohistochemistry}

The $4 \mu \mathrm{m}$ thick tissue sections were stained using the Bond-maX automated immunohistochemistry system (Leica Microsystems, Bannockburn, IL, USA). Antigen retrieval was performed using ER2 buffer (Leica Microsystems). Primary antibody against CD4 (1:100 dilution, Vision Bio-System/Leica Microsystems), CD8 (1:200 dilution, Novacastra/Leica Microsystems Inc.), Granzyme B (1:20, LabVision, Fremont, CA, USA), Perforin (1:20, LabVision), or T-cell receptor $\gamma / \delta$ (1:20, Endogen, Rockford, IL, USA) was incubated for $30 \mathrm{~min}$ at room temperature and the bound antibodies were detected using the bond polymer refine detection method (Leica Microsystems) following the manufacturer's protocol. For the remaining antibodies, $4 \mu \mathrm{m}$ thick tissue sections were stained using the DakoCytomation Autostainer (Dako, Carpinteria, CA, USA). Tissue sections were deparaffinized and endogenous peroxidase was blocked by treating with $3 \%$ hydrogen peroxide in absolute methanol for $30 \mathrm{~min}$. Sections were covered with normal horse or goat serum (Vector Laboratories, Burlingame, CA, USA) for $30 \mathrm{~min}$, primary antibody for 30 min, biotinylated secondary horse anti-mouse antibodies, or goat anti-rabbit antibodies (Vector) at 1:200 for $30 \mathrm{~min}$, strepatvidin-biotin-complex (Vector) for $30 \mathrm{~min}$, and diaminobenzidine (Dako) for $5 \mathrm{~min}$, then counterstained with hematoxylin for $15 \mathrm{~s}$. Antigen retrieval was performed with heat retrieval in EDTA buffer $\mathrm{pH} 8$ (Dako) for CD3 using the Decloaking Chamber (Biocare Medical, Concord, CA, USA); and with heat retrieval in citrate buffer $\mathrm{pH} 6$ (Dako) for TIA-1 (data not shown). No antigen retrieval was performed for CD20 and CD57. The following primary antibodies and their dilutions were used: CD3 (1:200, Dako), CD56 (1:50. Santa Cruz, Santa Cruz, CA, USA), CD57 (1:50, Novacastra), and TIA-1 (1:100, Abcam, Cambridge, MA, USA).

\section{Fluorescence in situ hybridization}

Nuclei from cytogenetic cell pellets from bone marrow were analyzed by fluorescence in situ hybridization. The MLL (mixed lineage leukemia) probe set was purchased from Vysis (Abbott Laboratories, Downer's Grove, IL, USA). The probes were applied to slides of the tumor cells and co-denatured at $75^{\circ} \mathrm{C}$ on an Isotemp 125D heat block (Fisher Scientific, Pittsburgh, PA, USA). Slides were incubated at $37^{\circ} \mathrm{C}$ overnight in a moist slide moat (Boekel Scientific, Feasterville, PA, USA). They were then washed in a $0.4 \times \mathrm{SSC}$ solution at $73{ }^{\circ} \mathrm{C}$ for $2 \mathrm{~min}$, followed by a 1-min wash in $2 \times \mathrm{SSC} / 0.1 \% \mathrm{NP}-40$ and counterstained with DAPI (Sigma, St Louis, MO, USA). Fluorescent signals from 100 to 200 cells were evaluated at $\times 100$ with a Nikon Eclipse E800 fluorescence microscope equipped with the proper filter sets. An Applied Imaging System (Santa Clara, CA, USA) was used to record images of representative cells.

\section{Statistical analysis}

Using an engraftment criterion of $>0.1 \%$ human CD $45+\mathrm{CD} 33+$ by flow cytometry to establish a biologically significant cutoff, bone marrow engraftment was considered as a binomial variable. The Fisher's exact test (Stata Verson 9.0, College Station, Texas, USA) was performed to test for association between FLT3 genotype (ITD and D835 considered in aggregate) and engraftment.

\section{Results}

\section{NSG mice show robust engraftment of primary human AML samples}

To study the engraftment of AML in NSG mice, we chose 35 samples representing a diverse group of AML classified according to the French-American-British and World Health Organization classification systems (Supplementary Table 1). ${ }^{11,12}$ Eight -10-week-old mice were sublethally irradiated 1 day before IV injection of 5-10 million mononuclear cells from 
samples. On average, samples were 90-95\% leukemic blasts. Mice were routinely analyzed 12 weeks after injection except where otherwise noted. A representative analysis of the gating strategy used to quantify viable human CD45+ CD33+ AML cells harvested from bone marrow and spleen of recipients is shown (Figure 1a). Bone marrow (Figure 1b, first panel) and spleen (Figure 1, second panel) cytospins stained with HEMA3 illustrate leukemic blast cells infiltrating hematopoietic organs of primary recipients (Figure 1b). H \& E staining of the spleen, liver, and kidney (Figure 1b, third, fourth, and fifth panels) shows additional sites of leukemic infiltration after robust NSG engraftment of numerous AML samples. Engrafted leukemic cells displayed morphological characteristics representative of the original French-American-British subtype injected, showing the expansion of the original leukemia and not an outgrowth of a leukemic subclone (data not shown). Low levels of peripheral blood engraftment were only seen in the high engrafters (data not shown). In contrast to earlier reports, no consistent correlation between spleen enlargement and efficiency of engraftment was observed in NSG mice among all samples tested. ${ }^{13}$ These results initially suggested that NSG mice engrafted with human AML samples would display features of the human disease.

To determine the quantitative level of engraftment, analysis of bone marrow, spleen, and peripheral blood was performed routinely by flow cytometry. The percentage of engraftment was determined as the number of cells expressing human CD45 and CD33 over the total number of viable cells harvested per mouse bone marrow or spleen multiplied by 100 (Figure 1c). AML samples were categorized as high ( $>10 \%)$, low $(<10 \%$ and $>0.1 \%)$, and non-engrafters $(<0.1 \%)$ based on the percentage of leukemic cells in NSG bone marrow as determined by flow cytometric analysis (Figure 1c; Supplementary Table 1). Mean engraftment percentages in the bone marrow per cohort of mice injected with AML samples show that significant levels of engraftment are achievable in a large number of samples tested, showing the robustness of engraftment of the NSG animal model in comparison to NS and NSB animal models historically used in these studies (Figure 1c).

Earlier studies have shown variability in time to tumor cell engraftment and suggest that prolonging the incubation time could increase the percentage of engrafting animals or the level of engraftment. ${ }^{14}$ Prolonging the incubation time of leukemic samples that displayed modest levels of engraftment at 12 weeks in NSG mice significantly enhanced the percent bone marrow engraftment in some samples (AML collections 878 and 391), but not others (AML collection 438) (data not shown). This shows that in contrast to other types of cancers, time to leukemic cell engraftment can vary significantly in a heterogeneous disease such as AML.

\section{Preferential engraftment of primary AML specimens with FLT3 mutations}

We next analyzed variables associated with engraftment. Samples expressing FLT3/ITD or an FLT3 D835 point mutation were analyzed to determine whether their expression correlated with increased leukemic cell engraftment in the NSG strain, as has been shown earlier in NS mice. ${ }^{15}$ A statistically significant difference $(P<0.05)$ between the engrafted and non-engrafted cohorts of AML samples based on FLT3 mutation status (Figure 1d) was observed. This result suggests that the presence of FLT3 mutations in AML correlates with an enhanced ability to engraft in NSG mice and is consistent with the enhanced engraftment capability of samples with FLT3 mutations observed in NS mice. ${ }^{15}$

Overall, these data show that, in contrast to leukemic engraftment in NS and NSB mouse models, robust engraftment of unsorted human AML mononuclear cells is readily achievable in the NSG mouse and thus provides a useful tool to study the effects of therapies in a leukemic model of AML representative of what is seen in patients. 


\section{Efficient secondary and tertiary engraftment of human AML into NSG mice shows the presence of leukemia-initiating cells}

Although improved engraftment of AML has been reported in NSG mice, earlier studies have introduced AML cells into newborn NSG primary and secondary recipients to determine the presence of leukemia-initiating cells. ${ }^{8}$ These studies are technically difficult and require large amounts of sorted CD34+CD38- AML cells (10 000-500 000) not always readily available from bone marrow aspirates. In addition, Taussig et al. as well as preliminary data (not shown) suggest that earlier sorting of cells with antibodies to antigens associated with leukemia-initiating cells can lead to antibody-mediated clearance of these populations and can mask the heterogeneity of SCID leukemia-initiating cells (SL-IC) within human AML cell populations. ${ }^{16}$ To determine whether AML from leukopheresis samples contain self-renewing leukemia-initiating cells, we harvested and pooled cells obtained from bone marrow and spleen of AML-engrafted primary recipient NSG mice and transferred 2.5 to 10 million unsorted human CD45+ CD33+ cells per secondary recipient mouse. As with primary recipients, engraftment was determined by daily monitoring for symptoms of disease or at 12 weeks when bone marrow and spleen engraftment of AML blasts was determined. As has been shown in NS mice, ${ }^{2}$ human AML engraftment into secondary NSG recipients showed the presence of self-renewing leukemia-initiating cells (Figure 2a). Mean engraftment levels were significantly higher in secondary than in primary recipients for the majority of samples analyzed, suggesting that NSG mice provide an environment that facilitates the growth or survival of leukemia-initiating cells. Engraftment from secondary into tertiary recipients retained robust levels of engraftment (Figure 2b). Leukemic engraftment was derived from the original leukemic clone as seen in an MLL translocation amplified from cells isolated from secondary (Figure 2c) and tertiary recipients (data not shown). These results suggest that serial transplantation retains the consistent engraftment of SL-IC and is not altered by multiple passages in mice. These results show that engraftment in secondary NSG recipients represents engraftment of SL-ICs and not passive transfer of leukemic progenitor cells.

\section{NSG mice represent an improved model for the expansion of primary human AML samples over earlier immunodeficient mouse strains}

Obtaining adequate amounts of leukemic cells for molecular and biochemical studies is challenging because of the limited access to viable and diverse leukemic samples. Earlier reports describing leukemic cell engraftment of NS and NSB mice have suggested that the expansion of leukemic cells was a notable characteristic of using these strains. ${ }^{1,2,6}$ However, in these studies, the expansion of leukemic cells was measured relative to the amount of cells present 2 days post-injection, which was determined to be 10-100-fold lower than numbers of cells initially injected, or was reported in comparison to the frequency of SL-ICs by limiting dilution analysis of unsorted or CD34+ CD38- sorted leukemic cells. Therefore, we next asked whether the NSG mouse model could function as a 'bioreactor' to expand human leukemic cell populations more efficiently than earlier immunocompromised animal models. Quantification of viable leukemic cells injected from AML leukopheresis samples compared with CD45+ CD33+ cell populations harvested from bone marrow and spleens from primary NSG recipients at 12-18 weeks indicated an average of up to 9-fold expansion of leukemic cells per recipient (Supplementary Figure 1) with up to a 16-fold expansion for some individual primary recipients. This shows that the NSG model is an improvement over earlier AML xenograft models because large quantities of human AML cells can be produced and isolated from these mice.

As mentioned earlier, engraftment variability among individual primary recipients was substantially reduced by engraftment into secondary recipients, with an overall increase in the percent of human CD45+ CD33+ cells engrafted among AML samples with robust NSG 
engraftment above $10 \%$. Human leukemic cell expansion in secondary recipients revealed up to a 23 -fold average expansion in comparison to primary recipients (Figure 2d). Leukemic cell expansion in multiple individual mice showed that up to a 44-fold expansion of leukemic cells was achieved. Cell expansion from tertiary recipients (data not shown) was comparable to secondary recipients. It is important to note that with some AML samples analyzed, partial spleens were collected and, therefore, our results are an under representation of the total leukemic cell expansion that can be achieved in these mice. Furthermore, leukemic cell isolation from mouse livers were not included in our analysis, and preliminary data (not shown) indicate that the liver can contain substantial numbers of leukemic cells and increase total leukemic cell expansion by up to 34-fold when taken into account. In conclusion, these results show that in addition to being a more robust model of leukemic cell engraftment, NSG mice provide a microenvironment for the expansion of leukemic cells and may be a valuable resource to obtain sufficient quantities of leukemic cells for molecular and biochemical analysis.

\section{Human leukemic cell engraftment is accompanied by the transient expansion of cytotoxic $T$ cells and fibrosis of NSG recipient bone marrow}

Despite robust cellular engraftment and expansion among some samples, not unlike earlier NS and NSB models, engraftment variability is seen among the different samples tested. We observed two biological phenomena that may contribute to the reduction in leukemic cell engraftment measured in select AML samples. First, we observed that femurs and tibias from heavily engrafted mice were white in appearance and when flushed consisted almost exclusively of pure leukemic blasts with a similar phenotype and morphological characteristics as the original sample. A representative bone marrow showing the complete replacement of marrow with leukemic infiltrate is shown in Figure 3a. However, in some samples, the abundance of leukemic cells observed at late time points post transplant caused necrosis of recipient bone marrows as shown in Figure 3b, an effect likely secondary to tumor microthrombi. There was progression to fibrosis as shown by $\mathrm{H} \& \mathrm{E}$ staining of cellular debris (Figure 3c) followed by reticulin fibrosis (Figure 3d). Advanced stages of bone marrow fibrosis as assessed by collagen staining (not shown) were also seen and described for the first time in a xenograft model of human leukemia, a pathological observation that is also seen in patients with advanced leukemia. ${ }^{17}$ As a result of bone marrow fibrosis, engraftment shifted to the spleen. However, despite increasing spleen engraftment, the overall leukemic cell yield from these mice was reduced because of loss of cells in the fibrosed bone marrow. The drop in bone marrow cellularity as seen with trypan blue exclusion was also reflected in a lower percentage of human CD45+ CD33+ cells on flow cytometry. Examples of this phenomenon are seen with AML samples 53 and 262 (Figures 1c and 2a, d; Supplementary Figure 1).

Second, we observed a minor population of CD45+ CD33- cells present in some leukopheresis samples used to inject primary recipients (Figure 4a, second panel). Analysis by flow cytometry revealed that this population was positive for the pan-T-cell marker CD2 (Figure 4a, third panel). In certain AML samples (listed by the asterisk in Supplementary Table 1), we observed the co-engraftment of human CD2+ cells with CD45+ CD33+ leukemic cells in the bone marrow and spleen of primary recipient animals (Figure $4 \mathrm{~b}$, third panel). In this representative animal, $\mathrm{CD} 2$ positive $\mathrm{T}$ cells expanded preferentially over CD45+ CD33+ leukemic cells (Figure 4b, second panel). We next wanted to determine the nature of the human $\mathrm{T}$ cells co-engrafting with the leukemic cells. Figure $4 \mathrm{c}, \mathrm{d}$, and $\mathrm{f}$ shows the presence of $\mathrm{CD} 3, \mathrm{CD} 8$, and Granzyme B positive cytotoxic T cells within the leukemic infiltrate in a spleen crosssection from a representative engrafted animal. Although CD57 staining for NK cells was also detected in the spleen (Figure 4e), additional surface markers for CD4, CD56, perforin, TIA-1, and TCR $\gamma / \delta$ (not shown) confirmed that these populations 
were atypical cytotoxic T cells and not NK cells. A minor population of CD19 positive B cells was also detected in the input leukopheresis samples and primary-engrafted recipients (Figures 4a and b); however, as they were not detected by histology, it is unlikely that they contributed a negative effect on the engraftment of leukemic populations in primary recipients.

To determine whether the presence of atypical human cytotoxic $\mathrm{T}$ cells in some leukemiaengrafted animals originated from normal hematopoietic stem/progenitor cells or from mature human T-cells present in the leukemia specimens, we compared the engraftment of a CD2-depleted primary leukopheresis sample at 12 weeks to the engraftment of human cells harvested from a non-CD2-depleted leukemia sample and transferred into a secondary recipient. Both the $\mathrm{CD} 2$-depleted and the undepleted samples passaged in secondary recipients engrafted with complete abrogation of the CD45+ CD33- cell population that was seen in the primary recipients (Figures $4 \mathrm{~g}$ and $\mathrm{h}$ ). These results suggest that there was a transient expansion of the patient's T cells in the leukopheresis sample and that the phenomenon was not caused by the development of $\mathrm{T}$ cells from engraftment of normal hematopoietic stem cells. As these cells were not stem cell derived, they were not capable of transfer to secondary recipients.

\section{Discussion}

Earlier immunocompromised mouse models of human AML have been a valuable tool for defining the biology of this disease, but have been limited in their ability to efficiently engraft leukemic cell populations and have not proven to be a robust model of human AML. The severe impairment of NSG innate immunity by targeted deletion of the IL2 receptor $\gamma$ common chain and the associated prolonged life span have made them an attractive model for long-term studies of the growth and evolution of both normal and leukemic cell development in vivo. This study presents the results of a comprehensive examination of primary human leukemia engraftment from a diverse group of AML specimens in the NSG mouse strain. The percentage of samples that engraft in NSG mice is comparable to the NS model and, similarly, engraftment is more likely for samples with an Flt3 mutation. ${ }^{15}$ However, in contrast to NS and NSB models, we observed a more robust leukemic cell engraftment and expansion in the bone marrow and spleen of recipient animals, as $40 \%$ of samples provided a high level ( $\geq 10 \%$ ) of bone marrow engraftment. This engraftment represented the expansion of true leukemia-initiating cells as engraftment levels and cytogenetic characteristics were retained in secondary and tertiary recipients. Interestingly, the disease phenotype observed in NSG mice recapitulates features of the human disease with evidence of necrotic and fibrotic bone marrow and infiltration of leukemic cells into non-hematopoietic tissues. Overall, these results provide a comprehensive description of a more robust model of human AML using the NSG mouse strain.

Several technical points should be emphasized about our results. In contrast to an earlier report describing AML engraftment in NSG mice, ${ }^{13,18}$ we observed low levels of leukemic cell engraftment in the peripheral blood only in the most robust engrafters. In contrast to the work by Ishikawa et al., ${ }^{18}$ we performed IV injections into adult (>8 week old) mice. It is possible that the use of adult mice as recipients alters the expression of chemokine receptors necessary to promote the movement of AML cells from the bone marrow into the peripheral blood. Engraftment analysis of samples described here using neonatal hepatic injection is underway and may clarify the critical variables in obtaining peripheral blood involvement with disease. We also noted that engraftment must be analyzed using both bone marrow and spleen. Although we have not performed sequential analysis, it seems that as the leukemia heavily infiltrates the bone marrow leading to fibrosis, the total cell yield and the level of AML engraftment in bone marrow may decrease, whereas the level of engraftment in the 
spleen increases. Thus, the bulk of the disease must be measured as total body load, not simply bone marrow involvement. Finally, we did not isolate leukemic cells from other mononuclear cells in preparing cells for injection and in a few cases observed engraftment of mature human $\mathrm{T}$ cells present in the leukemia graft. These cells have an atypical cytotoxic $\mathrm{T}$-cell phenotype and could be removed by T-cell depletion before injection in mice. These adoptively transferred mature $\mathrm{T}$ cells could also be eliminated from the leukemia graft by transfer into secondary recipients showing that they do not arise from a normal hematopoietic stem cell. Note that we observed T-cell engraftment at 12 weeks or more in $<10 \%$ of our samples suggesting that routine $\mathrm{T}$ cell depletion on all samples may not be necessary for these studies.

The significant difference between the NSG model and the NS model relates to the level of engraftment and expansion of AML cells in primary, secondary and, tertiary recipients. As noted, $40 \%$ of primary leukemia samples tested provided a high level of engraftment (over $10 \%$ chimerism) in the bone marrow. This increased level of engraftment represents a significant improvement over earlier immunocompromised strains and provides new opportunities for the development of new therapeutics for human AML. In addition, in some samples, we are able to increase the number of cells available for molecular or genetic studies.

Although this study represents a thorough examination of the engraftment of primary human human AML in NSG mice, differences between reported engraftment phenotypes compared with our data leave unresolved questions that the authors and others are actively investigating. Importantly, cumulative data from us and others show that the NSG mouse strain provides a substantially improved model for the study of human AML, both in the robustness of engraftment and expansion of leukemic cells, but more importantly, seems to model leukemic disease progression in human beings. This is tremendously important to the study of a disease that has with further characterization by gene expression, histone modification, and proteomics profiling, showed an immense heterogeneity between individuals and requires animal models in which to analyze disease progression in vivo.

\section{Supplementary Material}

Refer to Web version on PubMed Central for supplementary material.

\section{Acknowledgments}

We thank Catherine Keefer and Anthony Secreto of the Stem Cell and Xenograft Core at the University of Pennsylvania for their excellent technical assistance with NSG mice, leukemia cell injections, and animal health monitoring; Beth A Burke and Craig Jordan for critical evaluation of manuscript, design, and placement of figures; and Minu Samanta for analysis of FLT3 mutation expression in select AML samples. We also thank Neena J Panackal and Daniel Martinez from the Pathology Core Laboratories at the Children's Hospital of Philadelphia for processing mouse tissues and performing $\mathrm{H} \& \mathrm{E}$ stains on liver and kidney of engrafted recipients. $\mathrm{MC}$ was supported in part by the Leukemia Lymphoma Society of America.

\section{References}

1. Ailles LE, Gerhard B, Kawagoe H, Hogge DE. Growth characteristics of acute myelogenous leukemia progenitors that initiate malignant hematopoiesis in nonobese diabetic/severe combined immunodeficient mice. Blood. 1999; 94:1761-1772. [PubMed: 10477702]

2. Bonnet D, Dick JE. Human acute myeloid leukemia is organized as a hierarchy that originates from a primitive hematopoietic cell. Nat Med. 1997; 3:730-737. [PubMed: 9212098]

3. Lapidot T, Fajerman Y, Kollet O. Immune-deficient SCID and NOD/SCID mice models as functional assays for studying normal and malignant human hematopoiesis. J Mol Med. 1997; 75:664-673. [PubMed: 9351705] 
4. Christianson SW, Greiner DL, Hesselton RA, Leif JH, Wagar EJ, Schweitzer IB, et al. Enhanced human CD4+ T cell engraftment in beta2-microglobulin-deficient NOD-scid mice. J Immunol. 1997; 158:3578-3586. [PubMed: 9103418]

5. Shultz LD, Schweitzer PA, Christianson SW, Gott B, Schweitzer IB, Tennent B, et al. Multiple defects in innate and adaptive immunologic function in NOD/LtSz-scid mice. J Immunol. 1995; 154:180-191. [PubMed: 7995938]

6. Feuring-Buske M, Gerhard B, Cashman J, Humphries RK, Eaves CJ, Hogge DE. Improved engraftment of human acute myeloid leukemia progenitor cells in beta 2-microglobulin-deficient NOD/SCID mice and in NOD/SCID mice transgenic for human growth factors. Leukemia. 2003; 17:760-763. [PubMed: 12682634]

7. Shultz LD, Lyons BL, Burzenski LM, Gott B, Chen X, Chaleff S, et al. Human lymphoid and myeloid cell development in NOD/LtSz-scid IL2R gamma null mice engrafted with mobilized human hemopoietic stem cells. J Immunol. 2005; 174:6477-6489. [PubMed: 15879151]

8. Ishikawa F, Yoshida S, Saito Y, Hijikata A, Kitamura H, Tanaka S, et al. Chemotherapy-resistant human AML stem cells home to and engraft within the bone-marrow endosteal region. Nat Biotechnol. 2007; 25:1315-1321. [PubMed: 17952057]

9. Murphy KM, Levis M, Hafez MJ, Geiger T, Cooper LC, Smith BD, et al. Detection of FLT3 internal tandem duplication and D835 mutations by a multiplex polymerase chain reaction and capillary electrophoresis assay. J Mol Diagn. 2003; 5:96-102. [PubMed: 12707374]

10. Sheehan, D.; Hrapchak, B. Theory and Practice of Histotechnology. 2. St Louis: Mosby; 1980.

11. Bennett JM, Catovsky D, Daniel MT, Flandrin G, Galton DA, Gralnick HR, et al. Proposed revised criteria for the classification of acute myeloid leukemia. A report of the French-American-British Cooperative Group. Ann Int Med. 1985; 103:620-625. [PubMed: 3862359]

12. Vardiman JW, Harris NL, Brunning RD. The World Health Organization (WHO) classification of the myeloid neoplasms. Blood. 2002; 100:2292-2302. [PubMed: 12239137]

13. Agliano A, Martin-Padura I, Mancuso P, Marighetti P, Rabascio C, Pruneri G, et al. Human acute leukemia cells injected in NOD/LtSz-scid/IL-2Rgamma null mice generate a faster and more efficient disease compared to other NOD/scid-related strains. Int J Cancer. 2008; 123:2222-2227. [PubMed: 18688847]

14. Quintana E, Shackleton M, Sabel MS, Fullen DR, Johnson TM, Morrison SJ. Efficient tumour formation by single human melanoma cells. Nature. 2008; 456:593-598. [PubMed: 19052619]

15. Rombouts WJ, Blokland I, Lowenberg B, Ploemacher RE. Biological characteristics and prognosis of adult acute myeloid leukemia with internal tandem duplications in the Flt3 gene. Leukemia. 2000; 14:675-683. [PubMed: 10764154]

16. Taussig DC, Miraki-Moud F, Anjos-Afonso F, Pearce DJ, Allen K, Ridler C, et al. Anti-CD38 antibody-mediated clearance of human repopulating cells masks the heterogeneity of leukemiainitiating cells. Blood. 2008; 112:568-575. [PubMed: 18523148]

17. Kuter DJ, Bain B, Mufti G, Bagg A, Hasserjian RP. Bone marrow fibrosis: pathophysiology and clinical significance of increased bone marrow stromal fibres. Br J Haematol. 2007; 139:351-362. [PubMed: 17910625]

18. Ishikawa F, Yasukawa M, Lyons B, Yoshida S, Miyamoto T, Yoshimoto G, et al. Development of functional human blood and immune systems in NOD/SCID/IL2 receptor \{gamma $\}$ chain(null) mice. Blood. 2005; 106:1565-1573. [PubMed: 15920010] 


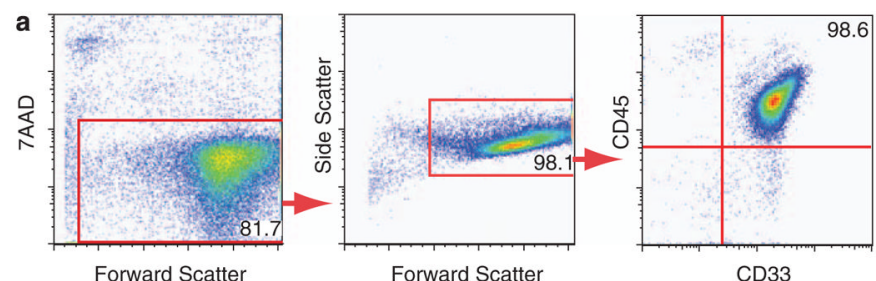

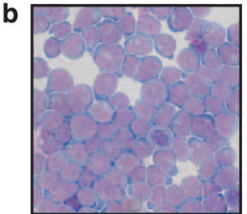

Bone Marrow 100x

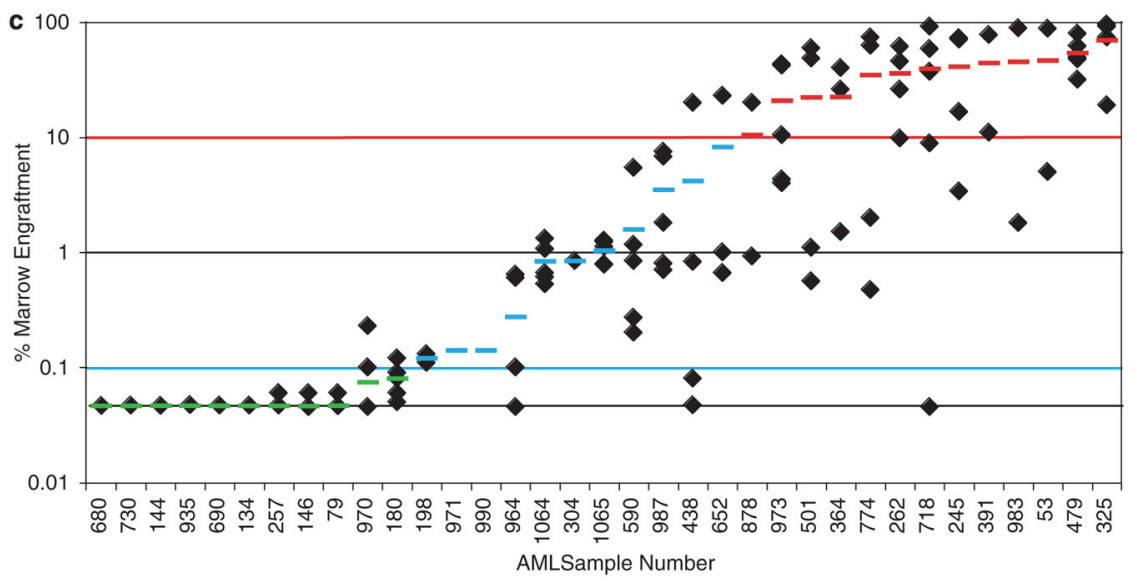

AMLSample Number

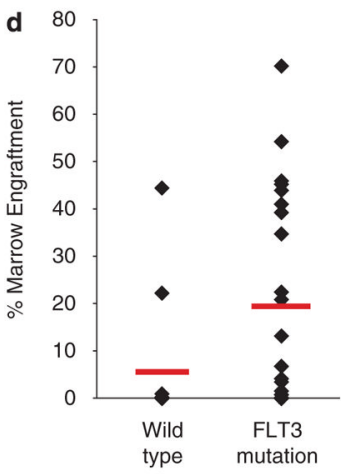

Figure 1.

Robust engraftment of primary human AML in NSG mice. (a) Representative flow cytometric analysis of bone marrow of an adult NSG mouse. 12 weeks after transplantation with $5 \times 10^{6}$ human AML cells injected through tail vein $24 \mathrm{~h}$ after sublethal irradiation. The bone marrow is completely replaced by CD45+CD33+ double positive cells consistent with human AML. (b) Cytospin with H \& E staining of bone marrow at $\times 100$. Cytospin with $\mathrm{H} \&$ E staining of spleen at $\times 50$. Spleen $H \& E$ staining at $\times 10$ with leukemic infiltrate. Liver $H$ $\&$ E staining at $\times 10$ showing sinusoidal pattern of leukemic infiltrate. Kidney $\mathrm{H} \& \mathrm{E}$ staining at $\times 10$, arrow shows a nest of leukemic cell infiltrate in the parenchyma of the kidney in a secondarily transplanted mouse. (c) Bone marrow engraftment of primary recipients $12-18$ weeks after transplantation. $5-10 \times 10^{6}$ human AML cells from thawed 
pheresis samples were injected through tail vein $24 \mathrm{~h}$ after sublethal irradiation. The bone marrow (two tibias and two femurs) was analyzed by flow cytometry for engraftment of human AML as shown in Figure 1a. The red line indicates a high level of engraftment of $>10 \%$ of murine bone marrow replaced with human AML. The blue line indicates the cutoff for engraftment. Samples with $<0.1 \%$ replacement of the bone marrow with human AML were considered to be non-engrafters. Each diamond represents a single mouse. The small lines indicated in green, blue, and red indicate the average for a given sample. The samples without diamonds were analyzed as pooled samples from five individual mice. The red highlighted sample numbers indicate samples that were serially engrafted and are shown in Figure 2; 13/35 samples showed a high level of engraftment (37\%), 10/35 showed a low level of engraftment (29\%), and 12/35 samples showed no engraftment (34\%). (d) Graphical representation of NSG engraftment (above $0.1 \%$ ) based on FLT3 wild type vs FLT3 mutated samples (ITD or D835 mutation). Statistical analysis determined a 95\% confidence interval of $P=0.03$ between engraftment of FLT3 wild type and FLT3 mutated samples. 

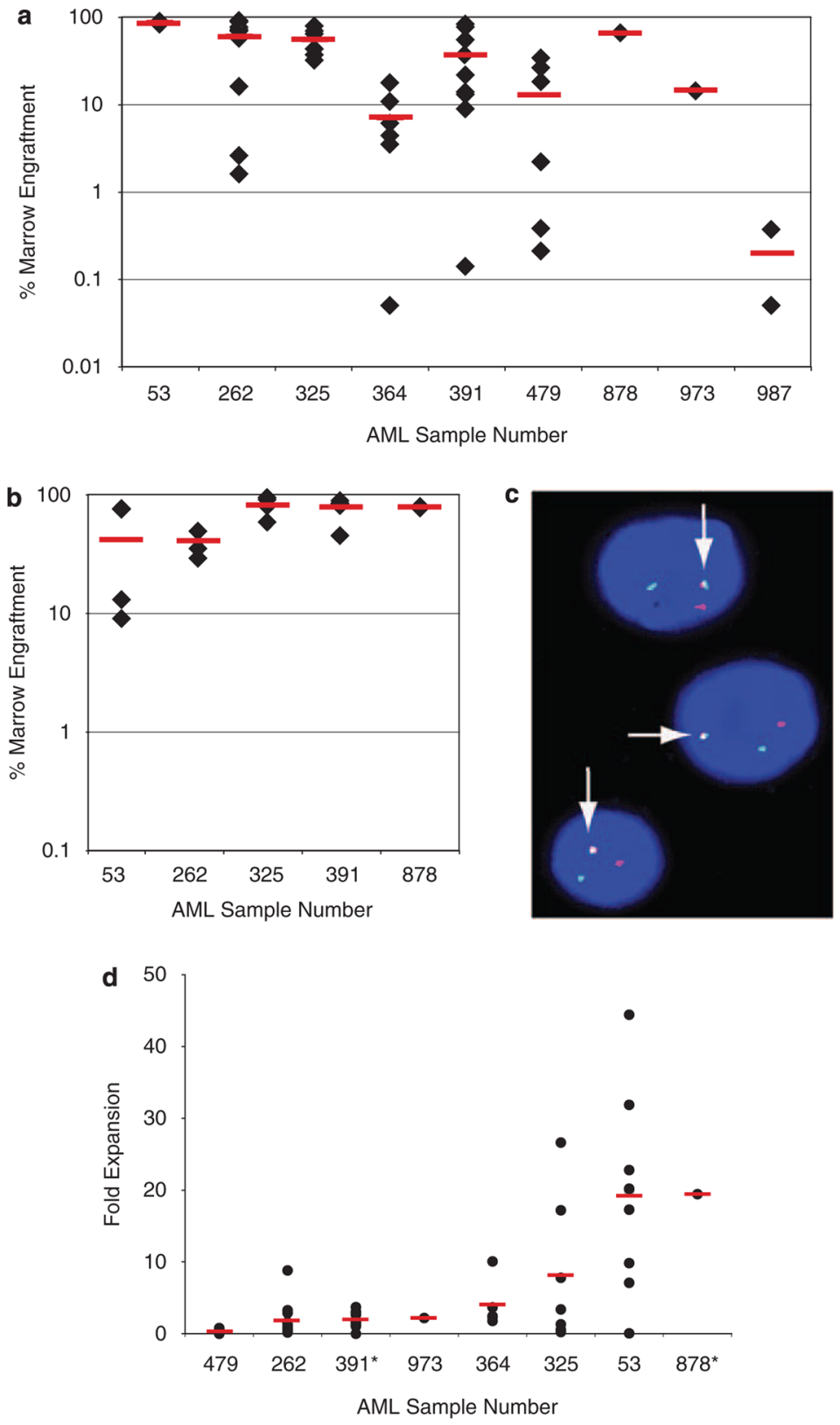

Figure 2.

Robust secondary and tertiary engraftment of human AML in NSG mice. (a and b) Bone marrow engraftment of secondary and tertiary recipients 12-18 weeks after serial passage; $5 \times 10^{6}$ human AML cells (pooled bone marrow and spleen cells) were injected through tail vein $24 \mathrm{~h}$ after sublethal irradiation. Each diamond represents a single mouse. The small lines in red indicate the average for a given sample. The bone marrow is replaced by CD45+ CD33+ double positive cells consistent with human AML. There is less variability in level of engraftment between mice with serial transplant. Heavily engrafted animals show a decrease in total bone marrow engraftment because of tumor necrosis in the marrow as shown in Figure 3. (c) Fluorescence in situ hybridization probe for MLL translocation of secondarily passaged sample 391 showed evidence of the MLL fusion in 100/100 cells tested, a representative picture is shown. (d) Human AML expands in secondarily 
transplanted mice as analyzed by flow cytometry $12-18$ weeks after transplantation. The red line indicates the average fold expansion per mouse $\left(10 \times 10^{6}\right.$ cells). Samples 364 had $6 \times 10^{5}$ cells injected per mouse. A black circle represents the total number of CD45+ CD33+ cells from an individual mouse analysis of four bones (two tibias and two femurs) and spleen. 
a

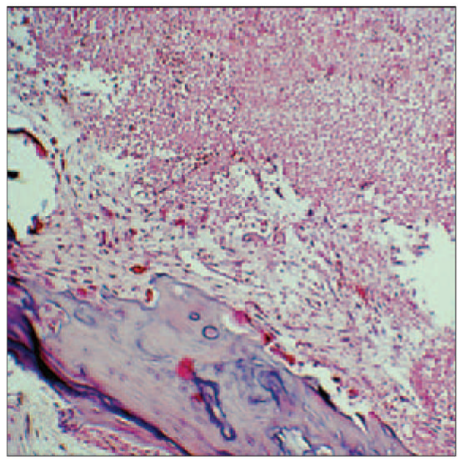

Bone Marrow 10x

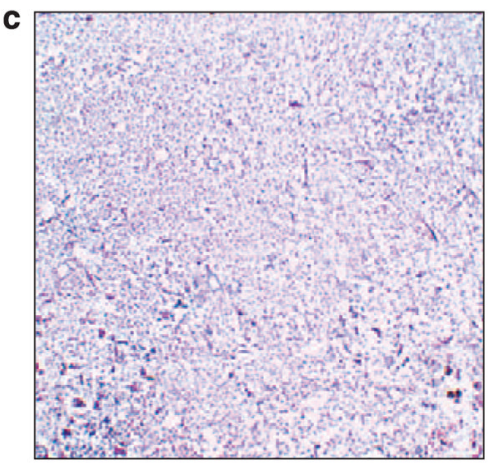

Bone Marrow 40x

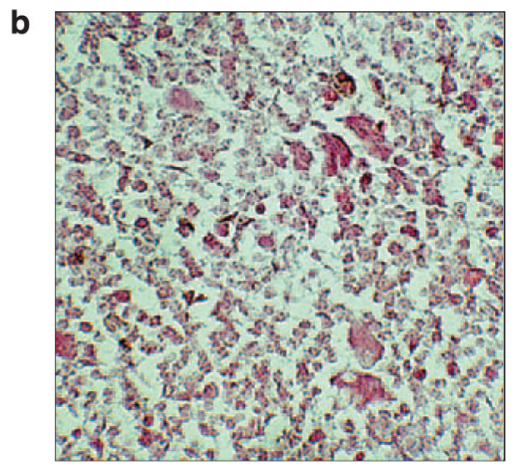

Bone Marrow 40x

\section{d}

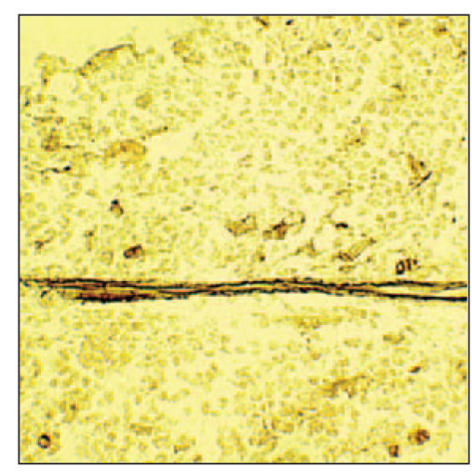

Bone Marrow 40x

Figure 3.

Leukemia-induced bone marrow fibrosis in NSG mice engrafted with human AML. (a) H \& E staining of femur shows complete bone marrow replacement with leukemic infiltrate at $\times 10$. (b) H \& E staining of bone marrow shows AML tumor necrosis at $\times 40$. (c) H \& E stain shows residual fibrous debris from tumor necrosis in the bone marrow at $\times 40$. (d) Reticulin staining shows reticulin fibrosis of the bone marrow at $\times 40$. Collagen fibrosis of the bone marrow by trichrome staining was seen, but is not shown. 

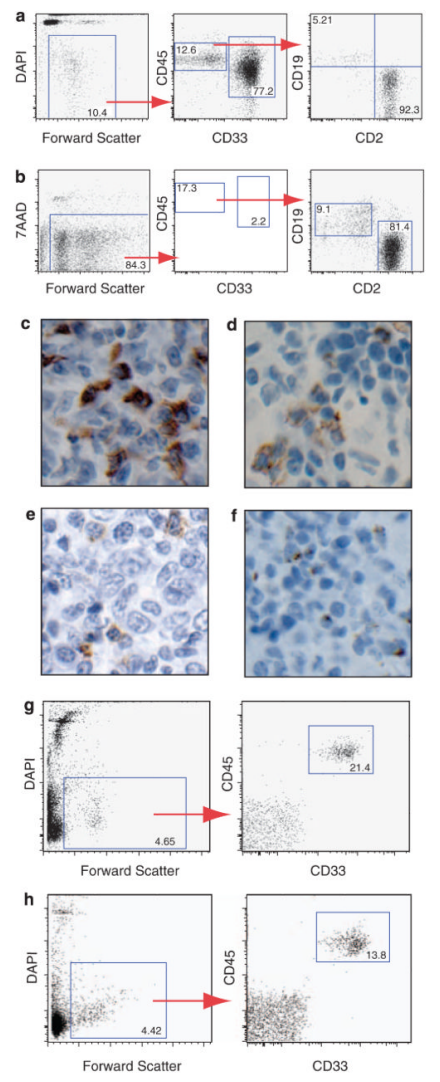

Figure 4.

Transient co-engraftment of human cytotoxic T and AML cells in NSG mice. (a) Flow cytometric analysis of primary sample 364 after thaw shows the presence of a CD45+ CD33- population in the pheresis sample. (b) Representative flow cytometic analysis of spleen of an adult NSG mouse engrafted with sample 364, 12 weeks after transplantation with $5 \times 10^{6}$ human AML cells injected through tail vein $24 \mathrm{~h}$ after sublethal irradiation. The spleen and bone marrow (not shown) is infiltrated by CD45+ CD33+ double positive cells consistent with human AML and a population of CD45+ CD33- cells consistent with cytotoxic T cells further characterized in (c-f). (c) Spleen CD3 immunohistochemical staining at $\times 40$ with leukemic infiltrate with scattered small and medium-sized cells staining positive for CD3 within the leukemic infiltrate. (d) Spleen CD8 immunohistochemical staining at $\times 40$ with leukemic infiltrate with scattered small- and medium-sized cells staining positive for CD8 within the leukemic infiltrate in similar pattern to CD3 staining. (e) Spleen CD57 immunohistochemical staining at $\times 40$ with leukemic infiltrate with scattered small- and medium-sized cells staining positive for CD57 within the leukemic infiltrate in a subset of the CD3+ cells. (f) Spleen Granzyme B immunohistochemical staining at $\times 40$ with leukemic infiltrate with scattered small- and medium-sized cells staining positive for Granzyme B within the leukemic infiltrate in a similar pattern to CD3 staining. These findings are representative of human cytotoxic T-cell infiltration of the human leukemic infiltrate in the murine spleen. (g) Secondary passage of whole bone marrow abrogates the CD45+ CD33- population of cells in the spleen and bone marrow (not shown). (h) CD2 depletion of the primary pheresis sample before primary engraftment also abrogates expansion of the CD45+ CD33- cell population in the spleen and bone marrow (not shown). 